\title{
CORRIGENDUM
}

\section{Effect of ion temperature on ion-acoustic solitons in a two-ion warm plasma with adiabatic positive and negative ions and isothermal electrons}

\author{
J. Plasma Phys., vol. 36, 1986, p. 301
}

\author{
By S. G. TAGARE
}

School of Mathematics, University of Hyderabad, Hyderabad 500134, India

(Received 12 December 1986)

In the above mentioned paper, table 1 is incorrect and the correct version is given below. Thus the result which was there obtained, namely that the coefficient of the nonlinear term of the modified $\mathrm{KdV}$ equation becomes negative, is not correct and, in fact, the coefficient is always positive.

\begin{tabular}{|c|c|c|c|c|c|c|}
\hline$Q$ & $\sigma$ & $n_{\beta c}$ & $A$ & $C$ & Amplitude & Width \\
\hline \multirow[t]{3}{*}{$0 \cdot 25$} & 0 & 0.0358 & 1 & $7 \cdot 3628$ & $0 \cdot 451$ & 1.414 \\
\hline & 0.025 & $0 \cdot 0191$ & 0.9170 & $20 \cdot 520$ & 0.282 & $1 \cdot 354$ \\
\hline & 0.050 & 0.8455 & 0.8455 & $66 \cdot 147$ & $0 \cdot 164$ & $1 \cdot 300$ \\
\hline \multirow[t]{3}{*}{0.476} & 0 & $0 \cdot 1140$ & 1 & $3 \cdot 0334$ & $0 \cdot 703$ & $1 \cdot 414$ \\
\hline & 0.025 & $0 \cdot 1016$ & $0 \cdot 9360$ & $4 \cdot 7147$ & 0.583 & $1 \cdot 368$ \\
\hline & 0.050 & 0.0873 & 0.8797 & $7 \cdot 3652$ & 0.232 & $1 \cdot 324$ \\
\hline \multirow[t]{3}{*}{0.887} & 0 & $0 \cdot 3092$ & 1 & $1 \cdot 1922$ & $1 \cdot 121$ & $1 \cdot 414$ \\
\hline & 0.025 & $0 \cdot 3256$ & 0.9964 & $1 \cdot 3914$ & $1 \cdot 062$ & $1 \cdot 383$ \\
\hline & 0.050 & $0 \cdot 3400$ & 0.9178 & 1.5918 & $1 \cdot 013$ & $1 \cdot 355$ \\
\hline \multirow[t]{3}{*}{1} & 0 & $0 \cdot 3660$ & 1 & 1 & $1 \cdot 225$ & $1 \cdot 414$ \\
\hline & 0.025 & $0 \cdot 3900$ & 0.9595 & $1 \cdot 1328$ & $1 \cdot 175$ & $1 \cdot 385$ \\
\hline & 0.050 & 0.4123 & 0.9240 & $1 \cdot 2978$ & $1 \cdot 136$ & $1 \cdot 359$ \\
\hline \multirow[t]{3}{*}{4} & 0 & $1 \cdot 2368$ & 1 & 0.2626 & $2 \cdot 390$ & $1 \cdot 414$ \\
\hline & 0.025 & $1 \cdot 3194$ & 0.9749 & $0 \cdot 2784$ & $2 \cdot 351$ & $1 \cdot 396$ \\
\hline & $0 \cdot 050$ & $1 \cdot 3983$ & $0 \cdot 9526$ & $0 \cdot 2912$ & $2 \cdot 325$ & $1 \cdot 380$ \\
\hline \multirow[t]{3}{*}{32} & 0 & 1.8794 & 1 & $0 \cdot 1797$ & $2 \cdot 922$ & $1 \cdot 414$ \\
\hline & 0.025 & 1.9752 & 0.9763 & $0 \cdot 1877$ & $2 \cdot 861$ & $1 \cdot 397$ \\
\hline & 0.050 & 2.0655 & 0.9550 & $0 \cdot 1983$ & $2 \cdot 814$ & $1 \cdot 382$ \\
\hline
\end{tabular}

